\section{Colonoscopic Treatment of Gallstone Ileus}

Gallstone ileus occurs almost exclusively in the elderly and accounts for $25 \%$ of mechanical small-bowel obstructions in patients over the age of 65 , with a mortality of $15 \%$ [1]. Surgery has been the treatment of choice, but has a mortality of $12-50 \%[1-4]$. We report here an instance of successful colonoscopic removal of a gallstone obstructing the ileocaecal valve.

A 91-year-old nursing-home resident with dementia was admitted as an emergency with a 1-week history of lethargy, anorexia, constipation and abdominal distension. She was dehydrated and was assessed as having a Glasgow Coma Scale score of 9/15, which improved with fluid resuscitation. Her abdomen was distended and tympanic, with obstructive bowel sounds. There were no palpable masses. She had significant cardiac morbidity, and laboratory tests showed a leucocytosis and an elevated urea concentration at $11.2 \mathrm{mmol} / \mathrm{l}$. A plain abdominal radiograph revealed grossly distended loops of large and small bowel. No air was seen in the biliary tree. No obstructing lesion was found on gastrograffin enema.

She was managed with nasogastric suction and intravenous fluids. Diagnostic colonoscopy was undertaken and a large gallstone was found to be impacted at the ileocaecal valve (Figure [1]). This was snared and retrieved using a large Olympus polypectomy snare. No other pathological abnormality was identified. The patient made a full recovery and was discharged back to the nursing home.

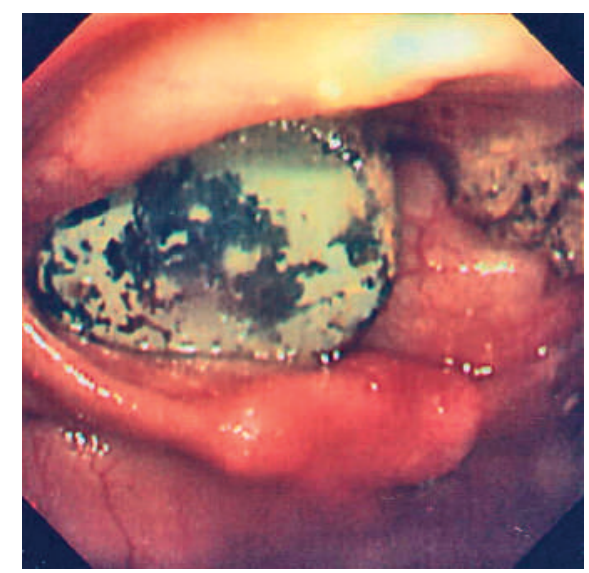

Figure 1 A gallstone impacted at the ileocaecal valve.

Early surgical intervention is the mainstay of treatment for gallstone ileus, regardless of the location of the stone [1]. Surgical treatment comprises enterolithotomy, with or without concurrent fistula repair and cholecystectomy. Both procedures carry significant morbidity and mortality [ $1-4]$. In this case, colonoscopy confirmed the site and cause of small-bowel obstruction and allowed definitive treatment in a patient who was a high surgical risk. Colonoscopic retrieval may be technically possible, carries small risks compared with surgery, and requires a shorter period of rehabilitation.

This paper supports the need for a trial of colonoscopic retrieval of a gallstone impacted at the ileocaecal valve. There is little to be lost from attempting endoscopic removal and, potentially, much to be gained. It is possible that there is a role for pre-procedure computed tomographic confirmation of the diagnosis.

Competing interests: Not declared

\section{E. L. Murray, M. Collie, \\ D. W. Hamer-Hodges}

Department of Colorectal Surgery,

Western General Hospital, Edinburgh,

United Kingdom

\section{References}

${ }^{1}$ Reisner RM, Cohen JR. Gallstone ileus: a review of 1001 reported cases. Am Surg 1994; 60: 441 - 446

${ }^{2}$ Clavien PA, Richon J, Burgan Set al. Gallstone ileus. Br J Surg 1990; 77: 737 - 742

${ }^{3}$ Deitz GM, Standage DA, Pinson CWet al. Improving the outcome in gallstone ileus. Am J Surg 1986; 151: 572-576

${ }^{4}$ Illuminati G, Bartolucci T, Leo Get al. Gallstone ileus: report of 23 cases with emphasis on factors affecting survival. Ital J Surg Sci 1987; 17: 139-325

\section{Corresponding Author}

\section{E. L. Murray, M.D.}

The Colorectal Unit (Wards 23 and 24)

Western General Hospital

Crewe Road South

Edinburgh EH4 2XU

UK

Fax: +44-131-527-1018

E-mail: emmalouisemurray@hotmail. com 